Revue des patrimoines

$6 \mid 2005$

Patrimoine en situation : l'Inventaire général entre histoire et prospective

\title{
Deauville : création et développement urbain
}

\section{Didier Hébert}

\section{(2) OpenEdition}

Journals

Édition électronique

URL : http://journals.openedition.org/insitu/8569

DOI : $10.4000 /$ insitu. 8569

ISSN : $1630-7305$

Éditeur

Ministère de la culture

Référence électronique

Didier Hébert, « Deauville : création et développement urbain », In Situ [En ligne], 6 | 2005, mis en ligne le 15 mai 2012, consulté le 19 avril 2019. URL : http://journals.openedition.org/insitu/8569 ; DOI :

10.4000/insitu. 8569

Ce document a été généré automatiquement le 19 avril 2019

\section{(c)}

In Situ Revues des patrimoines est mis à disposition selon les termes de la licence Creative Commons Attribution - Pas d'Utilisation Commerciale - Pas de Modification 4.0 International. 


\title{
Deauville : création et développement urbain
}

\author{
Didier Hébert
}

1 Le succès de Trouville, qui fonda sa réputation sur la pratique des bains de mer aux effets curatifs, entraîna tout au long du XIX ${ }^{\mathrm{e}}$ siècle une floraison de stations balnéaires sur les côtes de la Manche. Leur création reposait sur des opérations spéculatives, qui répondaient toutes à un même schéma: achat de terrains par une personne privée et création d'une société immobilière chargée du morcellement et de la vente des terrains à lotir, sous l'autorité de laquelle étaient exécutés les travaux de voirie et la construction des équipements balnéaires. Ce processus d'appropriation du littoral connut son apogée sur les côtes de la Manche au milieu du XIX ${ }^{\mathrm{e}}$ siècle, plus particulièrement sur la frange maritime du pays d'Auge, avec la création des stations de Cabourg en 1854 (sous l'impulsion de l'avocat parisien Henri Durand-Morimbau), de Villers-sur-Mer en 1856 (à l'initiative de l'architecte Félix Pigeory), d'Houlgate en 1858 (sous la direction de l'architecte Jacques Baumier) et de Deauville l'année suivante.

\section{La réalisation d'un projet ambitieux}

2 Erigée sur des terrains désertiques à l'écart d'un petit bourg rural niché sur le flanc nordest du mont Canisy, Deauville fut considérée, dès sa fondation, comme l'opération de promotion foncière la plus importante jamais réalisée sur la côte normande (fig. $\mathbf{n}^{\circ} \mathbf{1}$ ). 


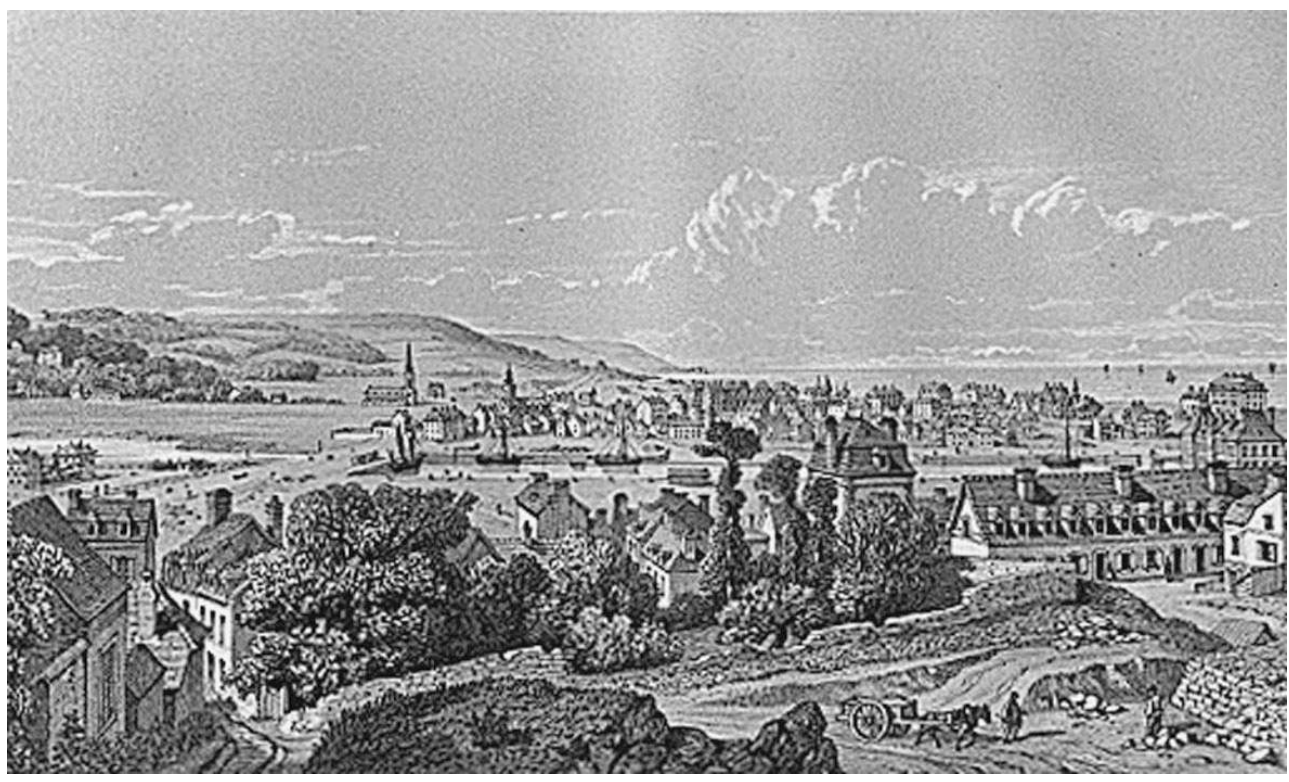

Deauville sous le Second Empire. Lithographie par Adolphe Maugendre, vers 1870, d'après un dessin de Théodore du Moncel. Musée municipal de Trouville [non coté]

Phot. Inv. F. Decaëns (c) Inventaire général, ADAGP, 2003

Ce jugement de valeur reposait autant sur la personnalité de ses fondateurs que sur l'ampleur du projet, comme en témoignent ces propos adressés par Henri Létang « aux spéculateurs et financiers » dans le Guide-Annuaire à Trouville-Deauville et aux environs de 1866 : «Vous qui cherchez une bonne opération, reposant sur des bases solides, avec la certitude de ne pas perdre vos capitaux, je vais vous indiquer une affaire unique [...]. Apprenez qu'il existe entre Pont-l'Evêque, Trouville et le vieux Deauville un espace plane, ayant 16 kilomètres de longueur, 4 kilomètres de largeur, parfaitement placé entre deux collines, un peu marécageux, arrosé par une petite rivière, la Touque, que la marée fait souvent déborder et dont une partie est envahie par les sables, traversé dans toute sa longueur par le chemin de fer de l'ouest [...]. La situation est admirable ; il ne faut, pour la rendre féconde, que l'intelligence, qui peut tout, et le capital, qui est la puissance sans laquelle on ne peut rien. Cette idée a été si parfaitement comprise par M. le duc de Morny, qui a vu dans la ville de Deauville [...] ce qu'il y avait à faire à cet emplacement, qu'il a obtenu du pouvoir le commencement de ce gigantesque projet, malgré l'opposition de tous les ports voisins, qui craignent et jalousent cette jeune ville adolescente, délicate, et qu'ils voudraient voir enterrée dans ses sables. Tout est réuni là pour faire une ville maritime de premier ordre [...]. Au point de vue d'un établissement de bains, d'un casino, d'une ville de plaisir, Deauville n'a à offrir que sa magnifique plage, qui existe partout sur le littoral et dans d'aussi bonnes conditions, et elle a pour rivale Trouville, la première des villes de bains de toute la Normandie, très aimée par tous les baigneurs [...]. Mais Deauville a l'utile: la place, l'espace nécessaire à toutes les grandes choses; son établissement n'est donc qu'une opération secondaire, ne pouvant être féconde que par son commerce. Ce qui est le réel, c'est donc son port, que l'on vient d'inaugurer, et qui n'est que l'avant-propos de cette immense entreprise créée par M. de Morny ».

Deauville ne fut donc pas conçue comme une simple station de bord de mer disposant de toutes les commodités d'une villégiature de luxe, mais comme une véritable ville, dotée 
d'infrastructures portuaires et ferroviaires. Elle devait ainsi être à même de dépasser le caractère économique des stations concurrentes, limité à la seule saison estivale, et de rivaliser avec le port du Havre. La légende véhiculée par les guides touristiques attribue la paternité de ce programme au duc de Morny, demi-frère de Napoléon III, président du corps législatif, détenteur depuis le début des années 1840 d'intérêts dans diverses opérations industrielles, financières et immobilières. Son rôle fut évidemment déterminant dans la bonne marche de l'entreprise, mais le véritable initiateur du projet fut en réalité son médecin personnel, Joseph olliffe, qui, pour assurer la réussite de son entreprise, s'effaça volontairement derrière l'aura du duc. Son mariage en 1841 avec l'Anglaise Laura Cubbitt, héritière d'une riche famille d'entrepreneurs de travaux publics et de constructeurs de chemin de fer, à qui l'on doit notamment les nouveaux quartiers de Bloomsbury et de Belgravia à Londres, avait assuré sa fortune. Propriétaire d'une villa depuis 1847 à Trouville, il ne fit, en créant la ville de Deauville, que reprendre à son compte et développer un projet initié par les édiles trouvillais en vue de l'extension du quartier résidentiel de la cité.

Figure 2

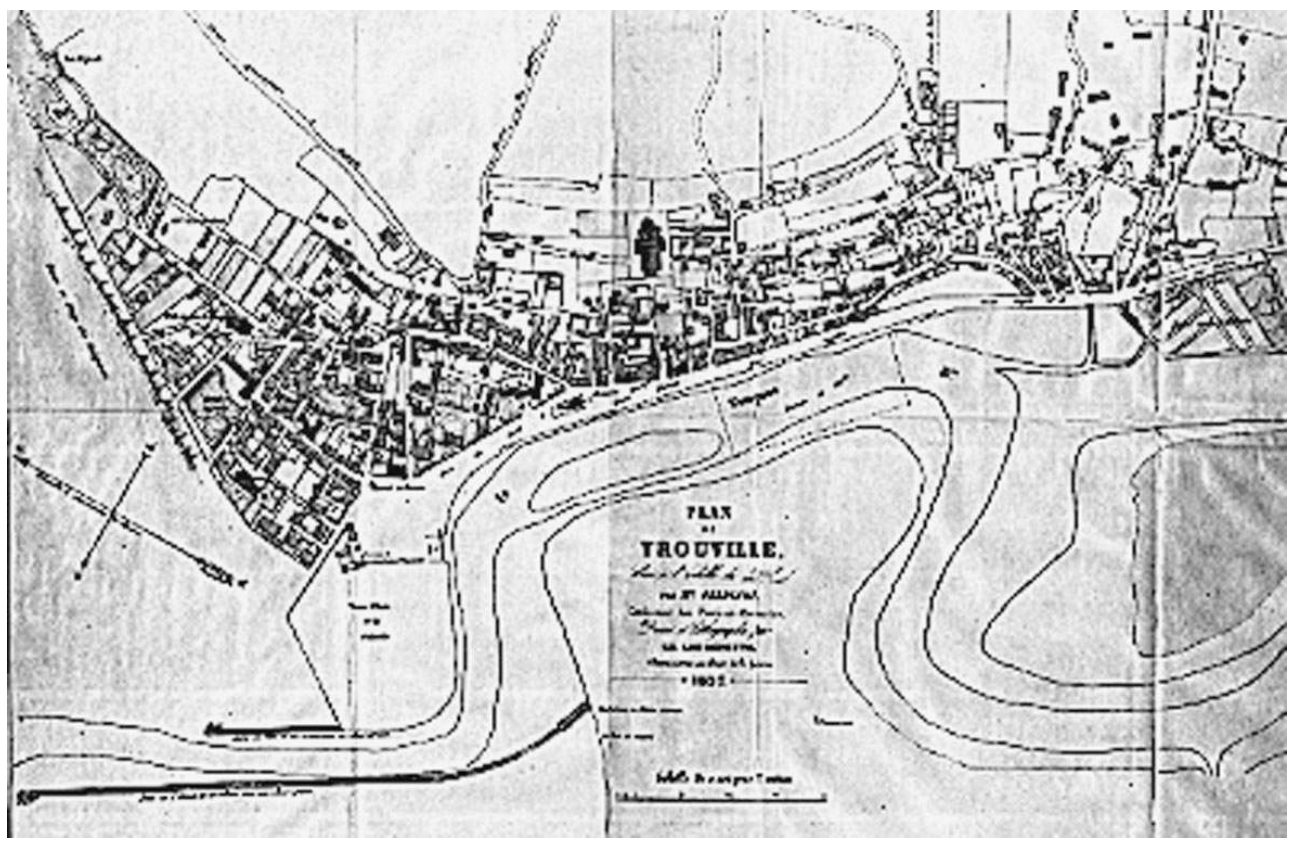

Plan de Trouville. Dressé par Jean-Louis Celinski de Zaremba, 1852. Musée municipal de Trouville [non coté]

Phot. Inv. P. Corbierre (C) Inventaire général, ADAGP, 1999

Depuis la découverte du site en 1825 par le peintre de marines Charles Mozin, Trouville

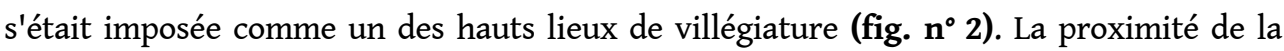
capitale, son caractère pittoresque et sa large plage de sable fin, plus attractive que celles de Haute-Normandie couvertes de galets, avaient été autant d'atouts pour séduire une élite parisienne en quête de dépaysement et soucieuse de cultiver une certaine hygiène de vie. Enclavé entre la Touques et la falaise des Roches Noires, à l'abri des vents marins, le bourg ancien avait rapidement été absorbé par les nouvelles constructions qui avaient bientôt conquis la presqu'île de la Cahotte, puis gagné le flanc ouest des Roches Noires. Echappant à toute règle d'urbanisme, la ville s'était structurée de manière spontanée, à partir d'initiatives privées, donnant lieu à un maillage de rues étroites et difficilement 
praticables, au tracé conditionné par l'implantation des bâtiments et le relief accidenté du site. Dès le début des années 1840 , l'exiguïté du territoire rendait impossible tout nouveau développement de la station. Aussi le comte d'Hautpoul, nommé maire en 1837, projeta-t-il de conquérir les terrains maritimes des communes limitrophes.

Figure 3

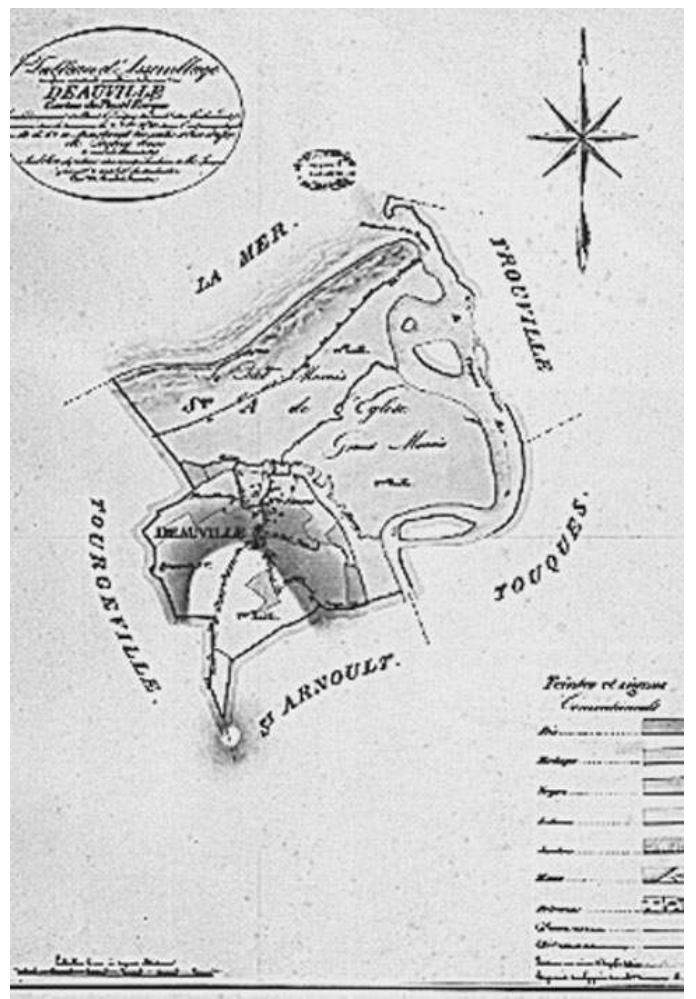

Cadastre napoléonien de la commune de Deauville. Plan d'assemblage dressé par Simon et Pescket, 1830. Archives départementales du Calvados, cote : 3P580

Phot. Inv. P. Corbierre (C) Inventaire général, ADAGP, 1998

En 1847, l'annexion du petit territoire d'Hennequeville permit ainsi d'étendre la plage de Trouville vers le nord-est. Son attention se porta également sur les marais de Deauville, vaste zone de landes s'étendant depuis le pied du mont Canisy jusqu'à la mer, où les habitants du coteau parquaient leur bétail et récoltaient le varech (fig. $\mathbf{n}^{\circ} \mathbf{3}$ ). En 1855 , il obtint du conseil d'arrondissement de Pont-l'Evêque un avis favorable au rattachement des deux communes, mais un litige sur la propriété des marais vint suspendre l'application de cette décision et la réalisation de ce second projet d'extension.

7 Le site de Deauville disposait d'un potentiel touristique considérable grâce aux moyens de communication développés sur la rive droite de la Touques, en direction de Pontl'Evêque, par une route départementale aménagée en 1840 ou du Havre, par un service de vapeur ouvert en 1853. Informé du projet du maire de Trouville et convaincu de pouvoir réaliser une opération fructueuse, Joseph olliffe décida d'acquérir lui-même les marais pour y fonder une nouvelle cité. Introduit dans les milieux financiers parisiens, il associa à son entreprise Armand Donon, héritier d'une riche famille de négociants-banquiers de Pontoise, consul général de l'empire ottoman, administrateur de la Caisse d'escompte et directeur de la banque Donon-Aubry-Gautier et Cie. La propriété des marais ayant été finalement reconnue à la commune de Deauville en 1859, Donon et Olliffe se portèrent 
rapidement acquéreurs de 240 hectares de terrains en bord de mer et d'une source d'eau vive sur le coteau, moyennant 800000 francs. Approuvé par arrêté préfectoral du 6 décembre 1859, ce marché mit un terme définitif aux rêves d'extension de Trouville. Quelques mois seulement furent nécessaires pour que sorte des sables une véritable ville, lieu privilégié des séjours estivaux de l'aristocratie parisienne. Ce caractère quasiinstantané de la naissance de la cité n'échappa pas à certains contemporains, tel C. James qui constata : « Lorsque l'année dernière me rendant à Deauville, j'aperçus de mon wagon une cité splendide s'élever là où peu de temps auparavant je n'avais vu qu'une steppe aride et sauvage, je me demandais si je n'étais pas le jouet de quelques illusions. Non, j'étais, au contraire, en face de la réalité. Les maisons étaient bien positivement des maisons, ces palais des palais, toute cette campagne une campagne véritable, tout au plus pouvais-je en conclure qu'on construisait aujourd'hui les villes à la vapeur $»^{1}$.

Pour commercialiser et valoriser les terrains à bâtir, les deux acheteurs fondèrent la Société des terrains de Deauville, à laquelle fut associé, sans que son nom n'apparaisse jamais, le duc de Morny. Celui-ci assura la promotion de l'opération auprès du groupe financier Morny-Delahante, qu'il dirigeait, et dont les activités s'appuyaient sur la banque Donon-Aubry-Gautier et Cie. Entre 1860 et 1866, pas moins de 80 lots furent vendus à de riches familles de propriétaires fonciers, tels Grehulle ou Sipière, à des banquiers, tels Tenré, Dalloz ou Boitelle, ou encore à des agents de change, tels Dollfus ou Salamanca. La création de Deauville ne fut donc pas simplement le fait de quelques individualités, mais de tout un groupe partageant des intérêts communs et qui consolidait ainsi son réseau d'influence $^{2}$. Les autres acquéreurs étaient directement liés au développement de la station: les entrepreneurs Castor et Mauger, chargés de réaliser les grands travaux, et l'architecte parisien Desle-François Breney, auteur du plan d'urbanisme.

\section{Inventer le territoire de bord de mer}

Sollicité par Joseph olliffe, pour lequel il avait réalisé en 1847 le casino-salon de Trouville, Breney fut chargé de concevoir l'organisation spatiale de la future cité. 


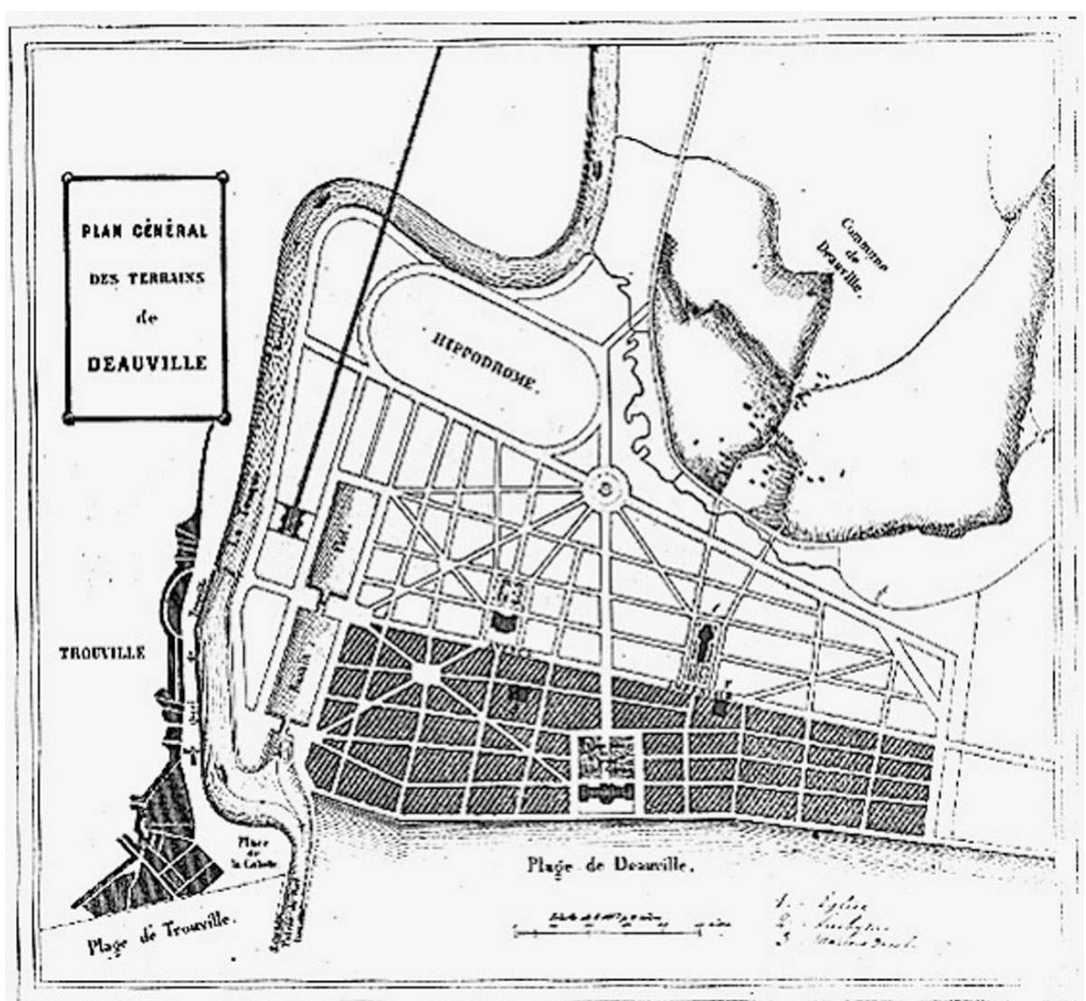

Projet du plan d'urbanisme de Deauville. Plan anonyme, vers 1859. Archives départementales du Calvados, cote : Fi 0989

Phot. Inv. P. Corbierre (C) Inventaire général, ADAGP, 1994

Il en dressa un plan, probablement vers 1859, au moment de l'acquisition des marais (fig. $\mathbf{n}^{\circ}$ 4), participant ainsi à la démarche «d'invention » du territoire de bord de mer, dont les principes avait été définis par l'architecte Paul Leroux lors de la conception du plan de Cabourg.

11 Breney dut s'adapter à un espace clos, bordé au nord par la mer, à l'est par la Touques, au sud par le coteau et à l'ouest par la commune de Tourgéville. Il délimita l'espace urbanisé par de larges boulevards, inscrivant la ville dans un trapèze. La composition d'ensemble reposait sur une structure géométrique en damier, avec des rues perpendiculaires et parallèles au rivage. Reprenant le tracé de la rue de la Mare (ouverte en 1790 afin de relier toutes les communes côtières depuis Dives-sur-Mer à l'ouest jusqu'à Honfleur à l'est), l'avenue Impériale délimitait deux grandes zones d'urbanisation que signifiaient des édifices majeurs :

- ouverte sur la mer, dont elle était séparée par une digue-promenade, une première zone se dégageait au nord. Formant le quartier résidentiel de la station, elle se déployait de part et d'autre d'un vaste jardin central, réservé au casino et aux grands hôtels de voyageurs. Ce dispositif était directement inspiré du plan de Cabourg.

- une seconde zone s'étendait depuis l'avenue Impériale jusqu'au coteau. Formant le quartier populaire de la cité, elle était structurée par deux places - l'une réservée à l'église, l'autre à la mairie -, aménagées de part et d'autre d'une artère allant du casino à l'hippodrome. 

(un avant-port et deux bassins éclusés) et d'une ligne de chemin de fer accueillait les activités industrielles et marchandes de la ville. Un pont, situé au débouché de la rue Impériale, et un bac, établi à l'embouchure de la rivière, assuraient la liaison avec Trouville.

Des axes fonctionnels, reliant les principaux édifices, assuraient l'articulation de ces trois secteurs. A la croisée des rues menant du bac à la mairie et de la gare au casino, était aménagée une place en étoile, l'actuelle place de Morny. Au sud-est, d'une place circulaire dont la composition en patte d'oie était héritée des parcs et jardins classiques, rayonnaient des artères distribuant l'église, le casino, la mairie et la gare. Le plan était complété par un ensemble de voies secondaires délimitant des îlots de surfaces inégales. Exclu du plan d'urbanisme, le bourg ancien était cependant relié à la ville nouvelle grâce au prolongement des chemins vicinaux dits de «Saint-Laurent » et de "Tourgéville à Deauville », qui encadraient la place de l'église.

\section{Figure 5}

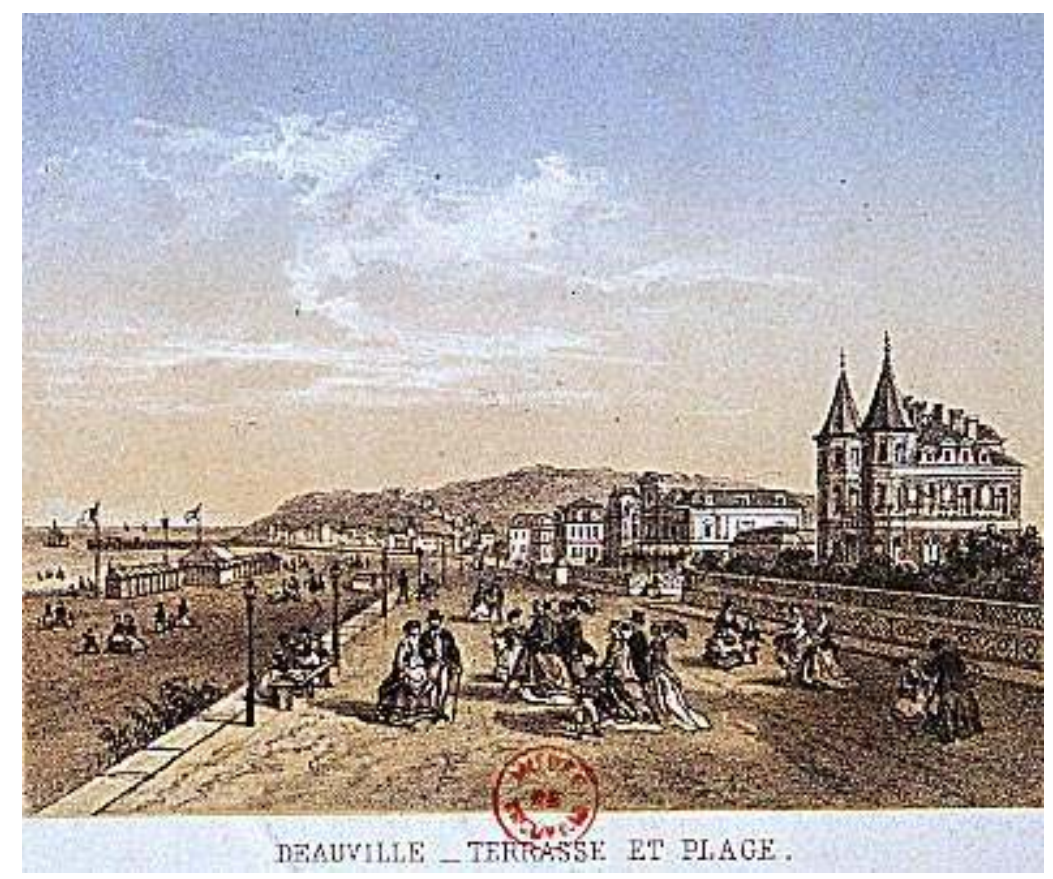

Deauville. Terrasse et plage. Lithographie anonyme, vers 1870. Musée municipal de Trouville [non coté]

Phot. Inv. F. Decaëns () Inventaire général, ADAGP, 2003

Breney proposa donc le plan d'une véritable ville, associant au quartier de bord de mer destiné à la villégiature, les espaces traditionnellement dévolus aux édifices publics et religieux et des équipements portuaires et ferroviaires devant permettre de développer le trafic marchand. L'architecte s'attacha également à intégrer les éléments urbains dans un cadre paysager. Cette mise en scène, compensant le manque de relief du site, prenait tout son sens depuis la plage, point de vue privilégié de la station (fig. $\mathbf{n}^{\circ}$ 5). Désireux de reproduire, à une échelle plus modeste, les principes d'organisation d'une ville moderne, Breney s'inspira des principes d'hygiène, d'esthétique et de circulation qui avaient régi les travaux parisiens d'Haussmann. Il recréa ainsi l'environnement connu par la clientèle 
visée afin que, selon l'expression de Pierre de Régnier, « la haute société se retrouve pour ainsi dire chez elle $»^{3}$.

Figure 6

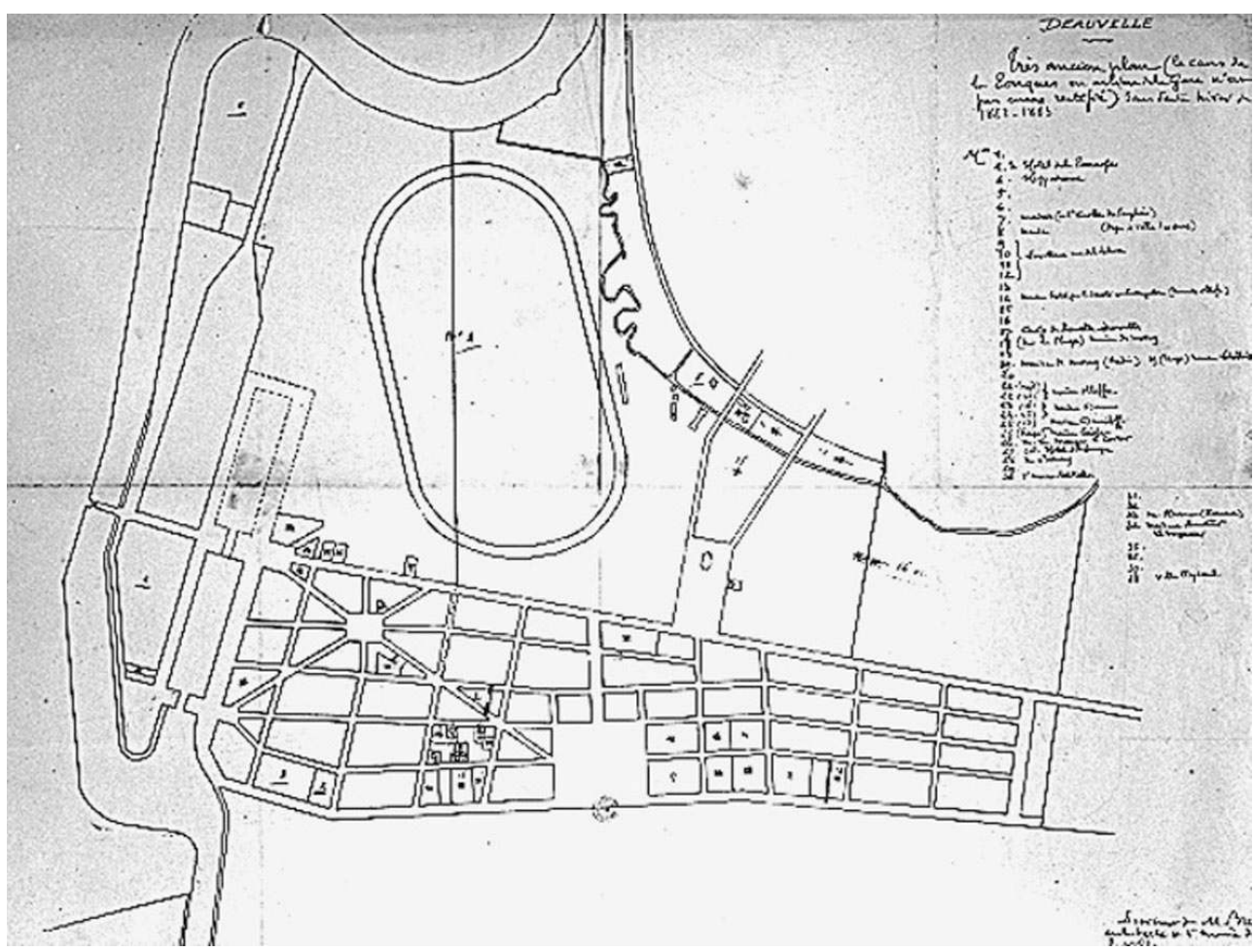

Plan de Deauville. Dessin par Henry Lecourt, d'après un plan dressé par Breney, vers 1860. Musée municipal de Trouville [non coté]

Phot. Inv. F. Decaëns (c) Inventaire général, ADAGP, 2003

Par cette rationalisation des espaces, le plan de Deauville s'affranchissait du plan empirique de Trouville. Sa composition en damier, courante en milieu urbain et reconnue pour sa facilité de mise en œuvre, permettait par ailleurs à la station de se démarquer de celle de Cabourg, célèbre pour son plan radioconcentrique. Le plan de Breney ne fut toutefois que partiellement réalisé. Hormis la place de l'église et le prolongement des chemins vicinaux depuis le bourg ancien jusqu'à la ville nouvelle, l'aménagement de la seconde zone fut en effet abandonné (fig. $\mathbf{n}^{\circ} \mathbf{6}$ ). Les véritables raisons qui motivèrent cette décision, prise probablement dès 1860 , au moment où les grands travaux furent engagés, ne nous sont pas connues. Le duc de Morny, grand promoteur des réunions hippiques, sut cependant tirer profit des terrains demeurés vierges, sur lesquels il fit aménager un hippodrome beaucoup plus vaste que celui initialement prévu. CEuvre de l'architecte Saint-Germain, celui-ci fut inauguré en août 1863. Les courses qui s'y disputèrent s'imposèrent dès lors comme l'événement sportif et mondain de la saison estivale.

\section{Les infrastructures portuaires et ferroviaires}

Le décret impérial du 25 juin 1860 autorisa le démarrage des grands chantiers: rectification du cours de la Touques, construction d'un pont sur la rivière, aménagement 
d'une ligne de chemin de fer et percement d'un bassin à flot. Symbole de l'alliance des stations de Trouville et de Deauville, le pont de l'Union fut inauguré en 1861.

Principal actionnaire des chemins de fer de l'ouest, le duc de Morny obtint sans difficulté l'autorisation de réaliser le prolongement de la ligne ferroviaire Paris-Lisieux-Pontl'Evêque jusqu'à Deauville. Véritable point d'appui du développement de la station, la gare donna lieu à un litige entre les deux stations rivales : elle avait en effet été implantée sur un ancien banc de sable encerclé par les méandres de la Touques, dont Trouville revendiqua la propriété. Sans attendre l'issue du conflit, réglé le 30 septembre 1863 au profit de Deauville, l'édifice, qui mettait Paris à cinq heures de la côte, fut inauguré le $1^{\text {er }}$ juillet.

Dès 1860, Donon et Olliffe avaient cédé au domaine les terrains nécessaires à la construction d'un avant-port éclusé et d'un bassin à flot. Ces aménagements portuaires devaient compléter les deux jetées aménagées entre 1846 et 1849 à l'initiative de la municipalité de Trouville pour fixer le chenal et faciliter l'accès à son port d'échouage. Large de 80 mètres, long de 300 mètres et disposant d'une cale de débarquement, le bassin à flot fut inauguré le $1^{\text {er }}$ août 1866 et relié, cette même année, à la ligne de chemin de fer par un embranchement ferroviaire. La "presqu'île de la Touques ", espace compris entre le bassin à flot et la rivière, fut alors investie par des usines spécialisées dans le traitement de la houille et du bois importés d'Angleterre et des pays du Nord.

\section{Les édifices identitaires de la cité}

Les équipements de villégiature furent réalisés entre 1860 et 1864, après que les marais eurent été asséchés, les dunes arasées et la voirie tracée. Cette dernière ayant été surélevée par rapport au niveau des marais d'une hauteur de 1,50 mètre, les terrains à bâtir se trouvaient en contrebas de la chaussée, ce qui contraignit les constructeurs à prévoir un étage de soubassement pour asseoir les villas et le remblai des terrains pour aménager les jardins. Destinés à donner l'impulsion aux constructions privées, les premiers édifices à sortir des sables furent les villas des fondateurs. 


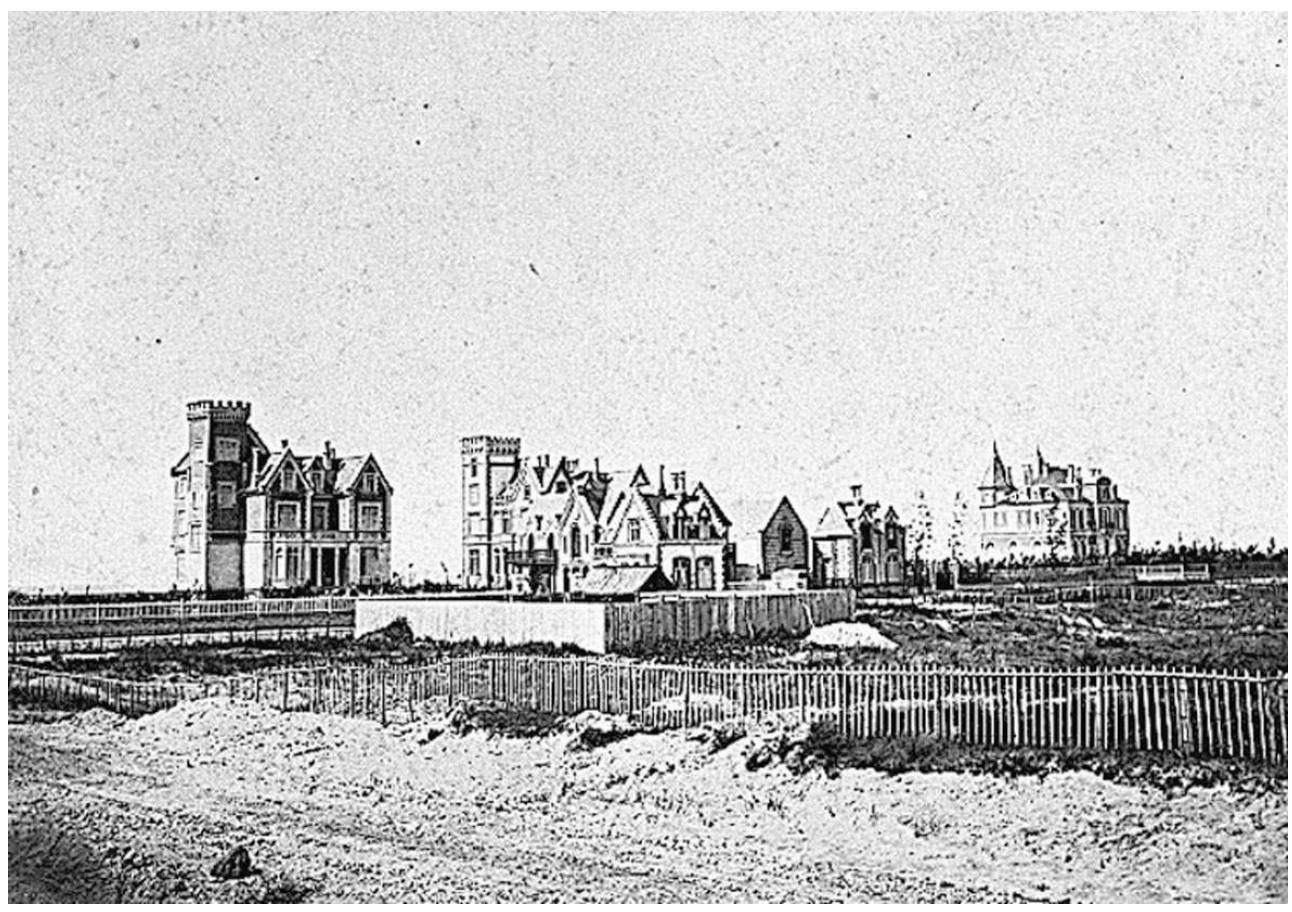

Les villas Victoria Lodge, Elisabeth et Sergewna. Cliché Eugène Villette, vers 1861. Musée municipal de Trouville [non coté]

Phot. Inv. P. Corbierre (C) Inventaire général, ADAGP, 1999

Construites sur de vastes terrains par Donon, Olliffe et Morny, les villas Elisabeth, Victoria Loge et Sergewna (fig. $\mathbf{n}^{\circ}$ 7) suscitèrent aussitôt l'intérêt des investisseurs, qui se pressèrent dans le marais pour investir les terrains les mieux exposés : ceux disposant d'un accès direct au rivage et d'une vue sur la mer. Aménagés en jardins d'agrément, ceux-ci furent occupés par de vastes demeures, dont le traitement architectural, ostentatoire et pittoresque, reflétait la réussite sociale et le goût de leur commanditaire. Ainsi, la ville offre «partout l'élégance, partout l'inattendu, partout la fantaisie dans ce qu'elle a de plus exquis et de plus ingénieux. Ici, un chalet russe aux délicates arabesques ; à côté, une maison hollandaise avec tour et pignon étayés; plus loin, deux villas construites dans le style le plus pur de l'ancienne architecture anglaise, plus loin encore et sur tout le profil de la terrasse, une série de créations fantastiques, réunissant toutes les variétés et tous les genres, réunissant même toutes les nationalités. C'est ainsi que Suisses, Espagnols, Anglais, Américains, Hollandais et même Turcs, réalisent par leurs excellents rapports le rêve si cher aux utopistes de la fraternité universelle $»^{4}$. Dans un souci d'ordonnancement, les constructions furent soumises à une réglementation imposant le retrait des bâtiments à 10 mètres de la digue-promenade. Tracée en 1861, achevée en 1864, celle-ci prit le nom de Terrasse. Longue de 1800 mètres, large de 20 mètres, elle repose sur une épaisse maçonnerie couronnée par une bordure en granite. Grâce à l'installation d'une usine à gaz, en 1861, elle fut sous le Second Empire l'une des rares voies à disposer d'un éclairage public. 


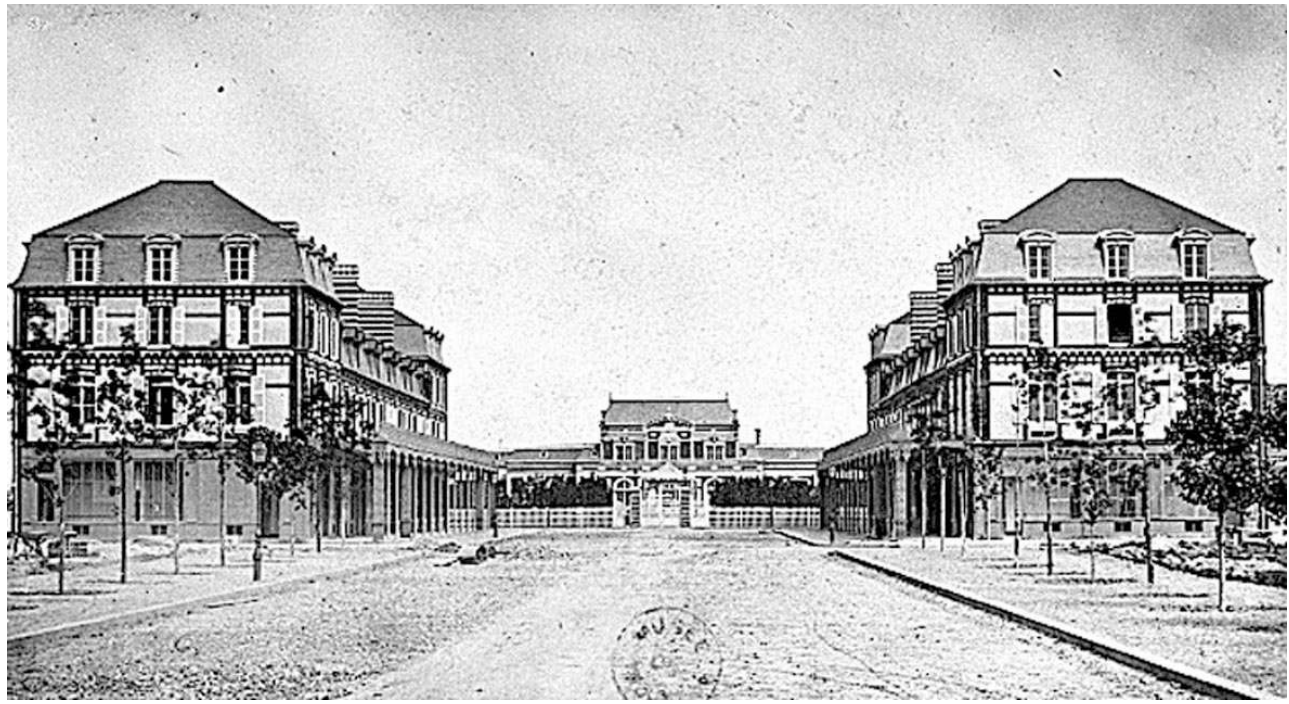

L'avenue de l'Hippodrome. Cliché Lepetit, vers 1870. Musée municipal de Trouville [non coté]

Phot. Inv. P. Corbierre @ Inventaire général, ADAGP, 1999 milieu d'un vaste parc de trois hectares aménagé dans l'axe central de la Terrasse. Privilégiant la composition au détriment de la vue sur la mer, l'architecte avait prévu de l'encadrer de deux hôtels de voyageurs perpendiculaires au rivage. Seul le Grand Hôtel du Casino fut cependant réalisé. A l'emplacement prévu pour le second hôtel, prit place en 1875 l'immeuble du Cercle, où se réunissaient les grands propriétaires d'écuries de courses de la station. Au sud-est, les «Arcades », deux immeubles symétriques abritant habitat de la villégiature à l'étage et magasins de commerce au rez-de-chaussée étaient construits de part et d'autre du casino. Ouvert sur l'avenue de l'Hippodrome, l'établissement de jeux était ainsi mis en valeur par la visée axiale, l'un des principes fondateurs en urbanisme (fig. $\mathbf{n}^{\circ} \mathbf{8}$ ).

Contrairement à Trouville, qui avait fondé sa notoriété sur les bains de mer, Deauville se voulait ville de plaisirs. Ses promoteurs veillèrent toutefois à ce qu'elle dispose des équipements nécessaires à leur pratique et la dotèrent, en 1862, d'un établissement hydrothérapique. Implanté à l'extrémité nord-est du front de mer, il dispensait des bains spécifiques à base d'eau chaude et d'eau froide, d'eau douce et d'eau de mer, et assurait la location et l'entretien des cabines de bains fixes ou mobiles, disposées sur la plage. $\mathrm{Ne}$ parvenant pas à détrôner Trouville dans ce domaine, il n'eut qu'une existence éphémère et fut détruit en 1877.

Devenu maire en 1861, Desle-François Breney favorisa certaines transactions avec la Société immobilière, dont il était l'un des principaux actionnaires. La municipalité prit ainsi en charge l'entretien de certaines rues en échange de terrains destinés à la construction d'édifices publics (deux sont cédés en 1864 pour l'église Saint-Augustin et le presbytère, trois autres en 1865 pour une école, le marché et, en prévision de l'arrivée massive d'une colonie anglaise, pour un temple), dont Breney et l'architecte parisien André Jal dressèrent les plans.

Dans le même temps, un quartier marchand s'organisa spontanément aux abords de l'actuelle place de Morny et le long de la rue du Casino. De modestes maisons et quelques 
rares immeubles, abritant au rez-de-chaussée des boutiques répondant aux besoins de la vie quotidienne, y prirent place à partir de 1863, le long de la voirie. Ces constructions laissaient entres elles de larges espaces qui restèrent inoccupés jusqu'à la fin des années 1890.

Le quartier populaire, où vivaient principalement des ouvriers du bâtiment, des commerçants, du personnel hôtelier ou des gens de maison, s'était développé sur les îlots attenants à la place de Morny, à proximité des équipements portuaires. Les habitations, maisons individuelles ou lotissements de quatre ou cinq logements, reprenaient les dispositions traditionnelles de la demeure urbaine, mitoyenne et alignée sur la rue. Chaque unité disposait d'un petit jardin et d'une remise en fond de parcelle.

\section{L'évolution du plan d'urbanisme}

Le décès du duc de Morny en 1865 et la chute du régime cinq ans plus tard affectèrent le développement de la station. L'aristocratie abandonna la plage de Deauville qui « devint déserte ; son magnifique promenoir, ses avenues, ses grandes rues, [furent] dépeuplées, inhabitées $»^{5}$. La crise économique de 1870 entraîna la faillite d'un certain nombre de scieries établies sur la presqu'île de la Touques. Le trafic marchand, concurrencé par le port du Havre, en sortit affaibli.

Figure 9

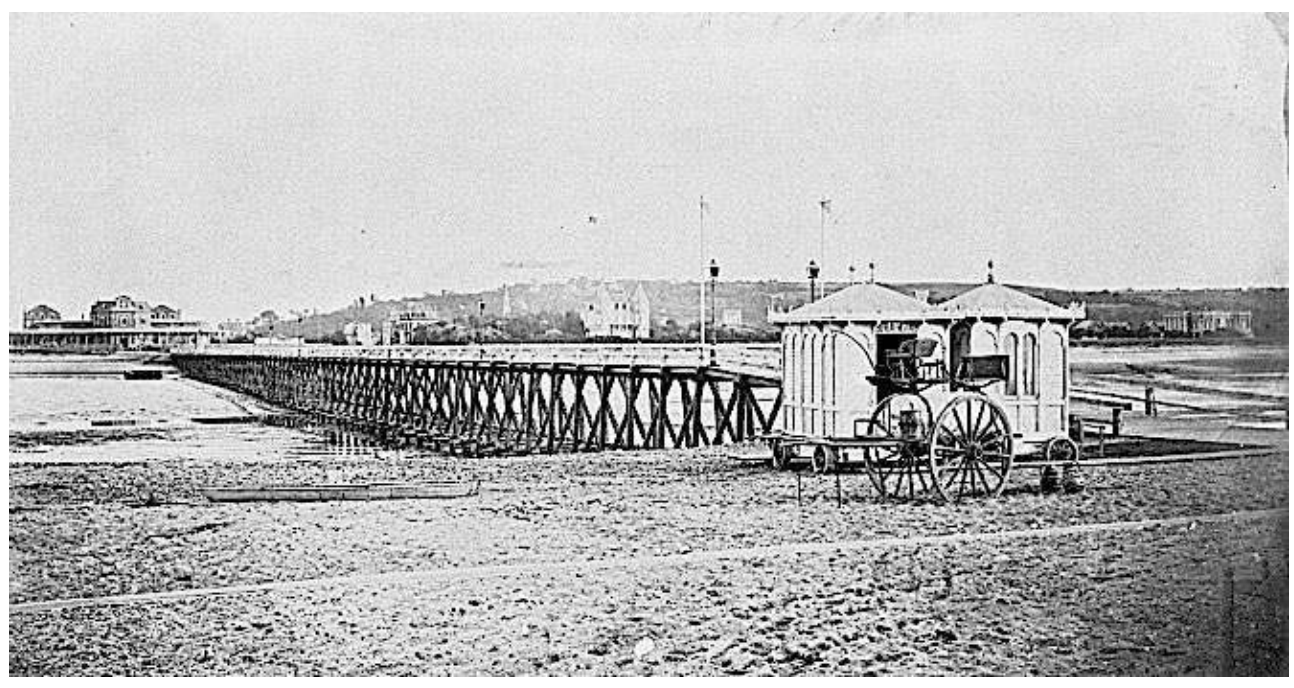

Jetée des bains de Deauville. Cliché Eugène Villette, vers 1875. Musée municipal de Trouville [non coté]

Phot. Inv. P. Corbierre (C) Inventaire général, ADAGP, 1999

A ces déconvenues politiques et économiques, vint s'ajouter une modification du bord de mer. La construction des jetées et les aménagements portuaires avaient en effet dévié les courants marins et favorisé les dépôts de sable sur la plage. Ce phénomène s'accéléra au cours de l'hiver 1874-75, suite à une violente tempête qui entraîna la formation d'un banc de galets à 300 mètres de la Terrasse, puis celle d'une noue. Pour atteindre le rivage, on dressa une estacade en bois, à l'extrémité de laquelle furent transférées les cabines de bains (fig. $\mathbf{n}^{\circ}$ 9). La société des bains de mer tenta de convertir la noue en lac et mit en place un système de sas pour permettre le renouvellement des eaux au moment du flux. 
Ces mesures se révélant insuffisantes, la noue fut progressivement comblée, créant ainsi une zone de lais de mer de 15 hectares. En 1883, le domaine en céda une partie aux propriétaires riverains, qui disposèrent ainsi de parcelles au droit de leur villa, avec interdiction d'y élever des constructions fixes ou d'y effectuer des plantations. La libre circulation du public y étant autorisée, les acquéreurs furent contraints de procéder au prolongement des rues perpendiculaires au rivage et, parallèlement à celui-ci, de faire aménager une terrasse empierrée de 10 mètres de large. En 1913, 17 nouveaux hectares furent cédés à la ville, à charge pour elle d'en rétrocéder une partie aux propriétaires riverains. Effective seulement en 1917, cette seconde cession ne comportait pas de clause de non aedificandi. Les parties privatives furent alors aménagées en jardin, tandis que la partie communale reçut des équipements sportifs et balnéaires (lawn-tennis en 1912, bains pompéiens et promenade des planches en 1924, Yacht-club en 1929). Les lais de mer devinrent ainsi le lieu privilégié des animations estivales de la station.

Après la chute du Second Empire, l'agglomération se densifia au rythme des périodes de dynamisme qui ponctuèrent l'histoire de la cité. Le cadre urbain tel qu'il avait été fixé par les fondateurs connut peu de modifications. Les bouleversements les plus importants s'opérèrent aux abords du casino, l'un des pôles majeurs de la cité.

En 1889, suite à des irrégularités constatées par le ministère de l'Intérieur, l'établissement de jeux ferma ses portes, privant la station de son lieu de sociabilité. Edmond Blanc, homme d'affaires et grand propriétaire d'écuries de courses, l'acquit quatre ans plus tard, avec le Grand Hôtel du Casino. Projetant d'aménager à cet endroit un lotissement de six villas, il fit détruire le casino en 1895 et percer la rue des Sports dans le prolongement de l'avenue de l'Hippodrome, ouvrant ainsi une perspective sur la mer.

Figure 10

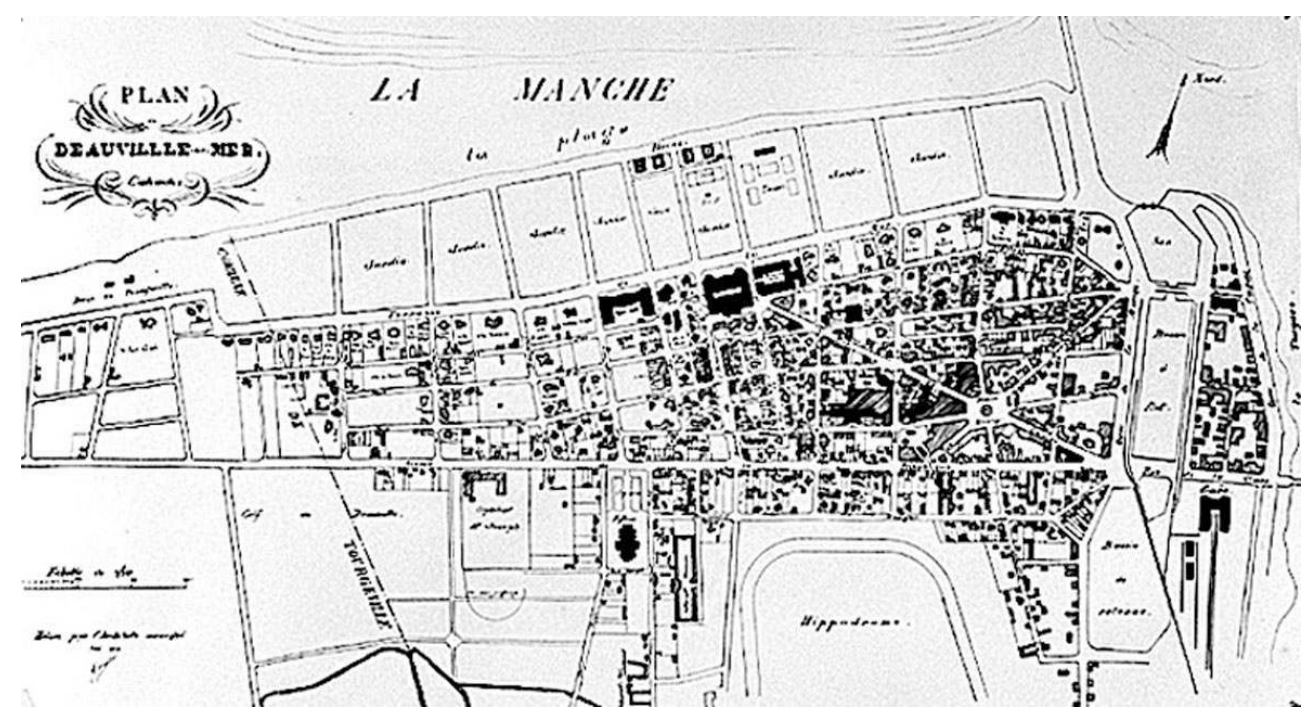

Plan de Deauville-sur-Mer. Plan anonyme, 1914. Archives communales de Deauville [non coté] Phot. Inv. F. Decaëns (C) Inventaire général, ADAGP, 2000

Au début du XXe siècle, Désiré Le Hoc, maire de Deauville, s'associa à l'homme d'affaires Eugène Cornuché pour relancer la station, en sommeil depuis 1870. Après avoir fondé la Société des casino et hôtels de Deauville, ce dernier se porta acquéreur en 1910 du Grand Hôtel du Casino et fit construire à son emplacement un nouvel établissement de jeux. Réalisé selon les plans de l'architecte Georges Wybo, l'édifice fut inauguré le 10 juillet 
1912. La même année, sur l'îlot attenant, prit place l'Hôtel Normandy, puis, l'année suivante, l'Hôtel Royal, à l'emplacement de la villa du duc de Morny détruite pour la circonstance (fig. $\mathbf{n}^{\circ} \mathbf{1 0}$ ). Face à ces édifices, sur les lais de mer, furent aménagés de vastes jardins d'agrément dessinés par l'architecte parisien Jean-Claude Nicolas Forestier, qui valurent dès lors à la station le nom de "plage fleurie ». Flattée par tant de luxe, auquel s'ajoutait la diversité des activités proposées (meetings aériens, courses automobiles, régate, golf et tennis), la riche clientèle cosmopolite se pressa à Deauville, qui connut alors un nouveau souffle.

En 1912-13, l'architecte Théo Petit érigea à l'arrière du casino un ensemble de boutiques de luxe, fournissant aux villégiateurs fortunés « tout le séduisant superflu indispensable à l'élégance ${ }^{6}$, et au centre duquel fut élevé le célèbre Café de la Potinière, réunissant à l'heure du thé les personnalités les plus prestigieuses de la cité. Cet ensemble fut complété par le magasin du Printemps, dessiné par Georges Wybo et Emile Mauclerc. Ces édifices constituèrent un nouveau noyau commercial, qui s'étendit progressivement jusqu'au centre-ville initial. Les terrains demeurés vacants aux abords de l'avenue du Casino furent investis par des immeubles occupés au rez-de-chaussée par des boutiques, les villas qui s'y trouvaient étant converties en magasin.

Les îlots réservés au quartier populaire s'avérèrent rapidement insuffisants pour accueillir une population en constante augmentation. Vers 1890, de nouveaux terrains à bâtir furent donc aménagés au-delà de l'avenue de la République (ancienne avenue Impériale), entraînant le percement du boulevard Mauger sur une partie des terrains de l'hippodrome. Aux abords du bassin à flot et du bassin de retenue, aménagés en 1889, s'organisa au début du XXe siècle un nouveau quartier populaire, constitué d'un habitat rudimentaire aligné sur une voirie de fortune. Cette zone offrant une image peu flatteuse de la station, la municipalité, à l'initiative de son maire Eugène Colas, mit en place une politique sociale sur le modèle des grandes villes industrialisées dans lesquelles se généralisaient les programmes de logements sociaux. En partenariat avec l'office départemental des habitations à bon marché, elle entreprit à partir de 1922 la réfection du quartier de l'Avenir, vaste lotissement en zone périphérique conçu sur le modèle des cités-jardins.

A la fin des années 1920, menacée par le succès des stations de la Côte d'Azur, Deauville chercha à renforcer ses infrastructures, mais se trouva confrontée à l'exiguïté de son territoire. Grâce aux revenus du casino, la municipalité finança alors deux programmes d'expansion sur les communes limitrophes. Elle acquit en 1927 des parcelles à Tourgéville et Bénerville pour y aménager l'hippodrome de Clairefontaine et acheta en 1930, sur la commune de Saint-Gatien-des-Bois, l'espace nécessaire à l'établissement d'un aérodrome. Les extensions extra muros ne furent toutefois pas le seul fait des pouvoirs locaux : sur la commune de Saint-Arnoult, François André, successeur d'Eugène Cornuché à la tête de la Société des casino et hôtels de Deauville, fit ainsi ériger en 1927 l'Hôtel du Golf, dotant ainsi la station d'un troisième palace.

\section{Conclusion}

Après la Seconde Guerre mondiale, la station connut des destructions massives, notamment sur le front de mer où les promoteurs remplacèrent de nombreuses villas du Second Empire par des immeubles résidentiels (fig. $\mathbf{n}^{\circ} \mathbf{1 1}$ ). 
Figure 11

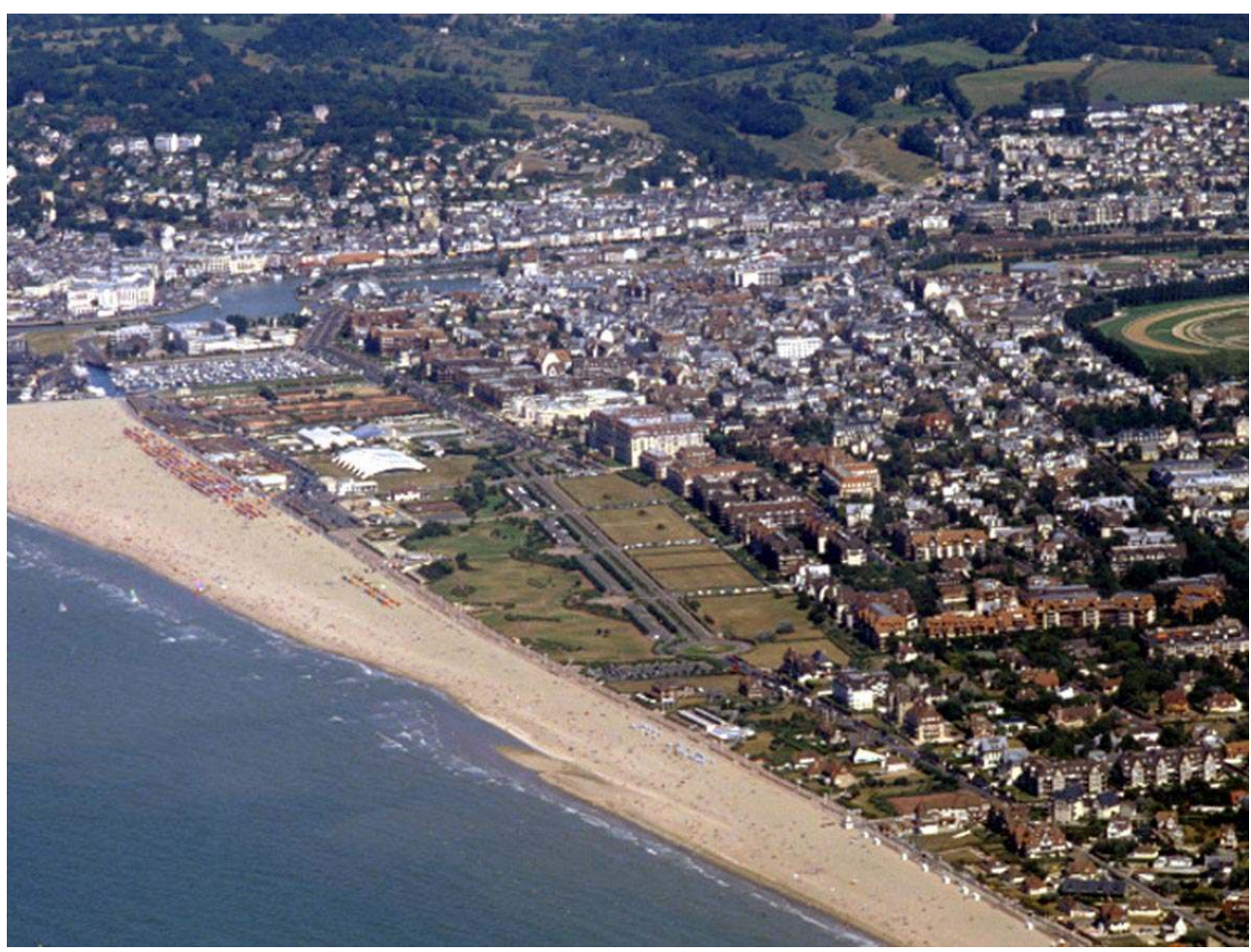

Vue aérienne du front de mer prise du nord-ouest

Phot. Inv. F. Decaëns (C) Inventaire général, ADAGP, 2003

Bien que ces constructions aient introduit une rupture d'échelle architecturale, les contours de la ville tels qu'ils avaient été définis par les fondateurs de la cité sont toujours lisibles. C'est en vue de sauvegarder ce patrimoine, participant de l'identité de la cité, que la municipalité, en partenariat avec les services du ministère de la Culture et de la Communication, a entrepris de mettre en place une Zone de protection du Patrimoine Architectural, Urbain et Paysager.

\section{BIBLIOGRAPHIE}

Chennebenoist, Jean. La côte fleurie à la Belle Epoque. Tome 1, Deauville, Bénerville, Blonville, Villers, Houlgate, Dives, Cabourg. Paris : Libro-Sciences, 1974.

Chennebenoist, Jean, Aublet, Yves. Deauville en Calèche. Deauville : Kaprika éditeur, 1983.

Culot, Maurice, Jakovljevic, Nada (dir.). Trouville. Bruxelles : Mardaga, I.F.A., 1989.

Culot, Maurice, Mignot, Claude (dir.). Deauville-Trouville : société et architectures balnéaires, 1910-1940 : la côte normande des années trente. Paris : Norma, I.F.A., 1992. 
Deliencourt, Roger, Chennebenoist, Jean. Deauville, son histoire. Tome 1 : Des origines à 1914. Honfleur : Marie, 1979.

Deliencouvert, Roger, Chennebenoist, Jean. Deauville, son histoire. Tome 2 : De 1914 à 1977. Honfleur : Marie, 1982.

Desert, Gabriel. La vie quotidienne sur les plages normandes, du Second Empire aux Années Folles. Paris : Hachette, 1983.

Garrigues, Jean. La République des Hommes d'Affaires 1870-1900. Paris : Editions Aubier, 1997.

Hebert, Didier. Deauville, une cité de villégiature de la Côte Fleurie. Cabourg : Editions

Cahiers du Temps, 2004 (coll. Images du Patrimoine, $\mathrm{n}^{\circ}$ 222).

Rouillard, Dominique. Le site balnéaire. Bruxelles : Mardaga, 1984.

\section{NOTES}

1. James, C. Guide pratique aux eaux minérales et aux bains de mer. Paris, [s.n.], 1867, p. 123.

2. Voir l'article de Sophie Cueille: Les stratégies des investisseurs : des bords de ville aux bords de mer. In Situ n 4, mars 2004, Les réseaux de la villégiature.

3. Regnier, Pierre de. Deauville. (Collection Portrait de la France). Paris : Editions Paul-Emile frères, 1927, p. 63.

4. Les bains de mer de Deauville. Guide, saison de 1868. Le Havre : imp. de Lemale, 1868, p. 24.

5. Simons, G. La baie de Seine, Le Havre, les côtes du Calvados. Paris, [s.n.], 1893, p. 256.

6. Guide Touristique, édité par le syndicat d'initiative de Deauville, 1912, p. 64.

\section{RÉSUMÉS}

Née de l'engouement de l'aristocratie du début du XIX ${ }^{e}$ siècle pour les bains de mer venus d'Angleterre et de la découverte de son site par des artistes à partir de 1825, la station de Trouville projeta sous le Second Empire de s'étendre sur les terrains inexploités de Deauville, sur la rive opposée de la Touques. Ces derniers furent acquis en 1859 par le docteur olliffe qui y fonda sa propre station, mettant un terme aux rêves d'extension de Trouville. Associant à son entreprise le duc de Morny, chef de file d'un puissant groupe de banquiers parisiens, il donna naissance à un projet séparatiste ayant pour but la spéculation foncière et la recherche du profit. Pour assurer le succès de leur opération, les promoteurs fondèrent la station sur un programme double: création d'un lieu de villégiature qui soit également un carrefour commercial doté d'infrastructures portuaires et ferroviaires. Ils confièrent l'élaboration spatiale de la future cité à l'architecte parisien Desle-François Breney. Adepte des principes haussmanniens, celui-ci s'efforça de concevoir une organisation territoriale distincte, par son ordonnancement, de la cité de Trouville. Partiellement exécuté, le plan du Second Empire est encore visible, malgré les percées réalisées à la fin du XIX ${ }^{\mathrm{e}}$ siècle, les extensions successives et la formation d'une zone de lais de mer qui offre à Deauville un front de mer singulier. 
During the nineteenth century, the success of Trouville, with its reputation founded on the novel practices of therapeutic sea bathing, gave rise to the development of seaside resorts all along France's Channel coast. Their creation was based on speculative initiatives which followed a similar general pattern: the purchase of real estate by a private speculator and the creation of a development company charged with dividing the land into parcels and commercialising the individual building plots. This company was also responsible for the layout of the resort's streets and for building its public seaside facilities. This process of appropriation of the Channel coast, notably on the maritime edge of Normandy's Pays d'Auge, was particularly dynamic during the middle of the century, with the creation of Cabourg in 1854 (under the impulsion of the Paris lawyer Henri Durand), Villers-sur-Mer in 1856 (at the initiative of the architect Félix Pigeory), Houlgate in 1858 (under the direction of the architect Jacques Baumier) and Deauville the following year.

\section{INDEX}

Keywords : sea bathing, seaside resort, Channel coast, Normandy

Mots-clés : Deauville, Trouville, bains de mer, balnéaire, Manche, bord de mer, Morny, opération immobilière, Joseph Olliffe, villa, villégiature, Normandie, Armand Donon, réseau d'influence, urbanisme, Desle, François Breney, Théo Petit, Georges Wybo, urbanisation, plan d'urbanisme, casino, hôtel de voyageurs, hippodrome, infrastructures portuaires, chemin de fer, lotissement, Eugène Cornuché, Georges Wybo, logements sociaux, ZPPAUP

\section{AUTEUR}

\section{DIDIER HÉBERT}

Inventaire. Région Basse-Normandie. didier.hebert@culture.gouv.fr 